\title{
TROPMET 97: An Overview
}

The TROPMET series of conferences on tropical meteorology was initiated by the Indian Meteorological Society (IMS) in 1992. Since then, TROPMET has provided an effective platform for the exchange of views and deliberations on a number of themes of scientific importance and national relevance. Today, with an average participation of a few hundred scientists cutting across a number of disciplines, TROPMET is a major national science event. An important aspect of the TROPMET series of conferences, inherited from its parent discipline, is its interdisciplinary character, encompassing atmospheric science, oceanography, fluid dynamics, numerical modelling, satellite technology and high-speed computing. 'TROMPET 97' was organized at Bangalore in February 1997 by the Bangalore Chapter of the Indian Meteorological Society on the theme 'Monsoon, Climate and Agriculture'. Despite the progress made during the last few decades in process modelling, numerical methods, high performance computing and better data acquisition, modelling of the Indian monsoon remains a challenge in terms of detailed simulation and prediction. Another major challenge is to understand and model associations between climate and weather variables and agricultural processes and to translate these for the benefit of farmers. Time was therefore considered to be ripe for critically examining the lessons learnt from the past experiences in monsoon modelling with a view to evolve appropriate strategies for meeting the challenges. 'TROPMET 97' witnessed a convergence of over 250 scientists representing a wide spectrum of disciplines.

The scientific presentations were organized in two parallel sessions, with a carefully chosen structure. The presentations covered the entire gamut of topics: modelling, observation and forecasting of monsoon, climate and agriculture. The scientific programme was divided into 14 sessions. Out of 180 contributed papers received, about 110 were presented orally while the rest were presented in poster sessions.

K S Yajnik

President, IMS, Bangalore Chapter.
B N Goswami

Secretary, IMS, Bangalore Chapter. 